\section{Gemella haemolysans: a rare and unusual cause of infective endocarditis}

Sir,

Bacterial endocarditis is a serious complication of cardiac disease with a mortality of between $10 \%$ and $20 \%{ }^{1}$ Mitral valve prolapse has been reported as the underlying cardiac abnormality in $10 \%$ of cases. ${ }^{2}$ We report a case of endocarditis caused by Gemella haemolysans in a 74 year old man with mitral valve prolapse. We believe this to be the first case reported in the United Kingdom.

A 74 year old man diagnosed 10 years previously as having mitral regurgitation secondary to mitral valve prolapse was admitted with a 2-month history of nonspecific myalgia and headaches. For 2 weeks he had had night sweats and rigors. He had not undergone any invasive procedures. On examination his temperature was $38.5^{\circ} \mathrm{C}$. There was a faint macular rash on both legs and signs of mitral regurgitation. Echocardiography showed prolapse of the posterior leaflet of the mitral valve with a small mass attached suggestive of a vegetation. A Gramvariable coccus was isolated from all 6 blood cultures and endocarditis of the mitral valve was diagnosed.

Identification of the isolate was attempted using the API 20 Strep system and this suggested Gemella haemolysans or Streptococcus morbillorum. It was confirmed as G. haemolysans by the National Collection of Type Cultures, Colindale. The organism was sensitive to penicillin (minimal inhibitory and bactericidal concentrations of $0.015 \mathrm{mg} / \mathrm{l}$ and $0.03 \mathrm{mg} / \mathrm{l}$ respectively) and he was given intravenous benzyl penicillin and intravenous gentamicin daily. He became apyrexial within 48 hours and remained so. After 2 weeks the penicillin was replaced by oral amoxycillin for 4 further weeks and the gentamicin given intramuscularly for 2 further weeks. Following cessation of antibiotics he continued to remain apyrexial, with negative blood cultures.

Mitral valve prolapse is well recognized as predisposing to infective endocarditis. In this case the organism responsible for the infection, $G$. haemolysans, is most unusual. The organism is considered to be a commensal of the upper respiratory tract. The exact taxonomy of this species is disputed but $\boldsymbol{G}$. haemolysans is related to the Streptococci and is biochemically similar to Streptococcus morbillorum, ${ }^{3}$ an organism which has also been reported as a cause of endocarditis. ${ }^{4}$ Infections caused by $G$. haemolysans were unknown until 1978-9 when three cases of endocarditis were reported in France. ${ }^{5}$ There have been further reports of Gemella infections from France, including two cases of septicaemia and a further case of endocarditis. ${ }^{6}$ A case of post-neurosurgical meningitis caused by $G$. haemolysans was reported from Oxford in $1985 .^{7}$ The strains isolated in all of the reported cases were highly sensitive to penicillin and synergy between penicillin and gentamicin has been demonstrated. ${ }^{8}$ In this case the infection rapidly responded to antibiotic therapy and the patient continues to remain well with no deterioration of his mitral valve disease.

\section{Acknowledgement}

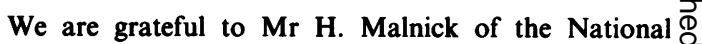
Collection of Type Cultures, Public Health Laboratory Service, Colindale, for the confirmation of the identity of ${ }^{\infty}$ this organism.

\section{M.J. Brack $\vec{\omega}$ P.G. Avery P.J.B. Hubner \\ R.A. Swann' 13 Regional Cardiothoracic Unit, Groby Road Hospital, 그 Groby Road, $\infty$ Leicester LE3 $9 Q E$ and iv 'Department of Microbiology $\overrightarrow{0}$ and Public Health Laboratory, ف่ Leicester Royal Infirmary, 음 Infirmary Road, Leicester, UK. $\rightarrow$}

\section{References}

1. Baron, D.W. \& Hickie, J.B. Changing concepts in the manage ment of infective endocarditis. Med J Aust 1977, 1: 767.

2. Corrigall, D., Bolen, J., Hancock, E.W. \& Popp, R.L. Mitre्9 valve prolapse and infective endocarditis. Am J Med 1977, 6. 215-222.

3. Berger, U. \& Pervanidis, A. Differentiation of Gemella haemolysans from Streptococcus morbillorum. Zbl Bakt Hyg 1986, 261: 311-321.

4. Maxwell, S. Endocarditis due to Streptococcus morbillorum. $J$ Infect 1989, 18: 67-72.

5. Chatelain, R., Croize, J. Rouge, P. et al. Isolement de Gemella haemolysans dans trois cas d'endocardites bactériennes. Med Mal Infect 1982, 12: 25-30.

6. Laudat, P., Cosnay, P., Icole, B., Raoult, A., Raynaud, P. \& Brochier, M. Endocardite à Gemella haemolysans: une nouvelle observation. Med Mal Infect 1984, 14: 159-161.

7. Mitchell, R.G. \& Teddy, P.J. Meningitis due to Gemella haemolysans after radiofrequency trigeminal rhizotomy. J Clin Pathol 1985, 38: 558-560.

8. Buu-Hoi, A., Sapoetra, A., Branger, C. \& Acar, J.F. Antimicrobial susceptibility of Gemella haemolysans isolated from patients with subacute endocarditis. Eur J Clin Microbiol 1982 14: $159-161$.

\section{Chlorhexidine-induced gastritis}

Sir,

Acute gastritis is caused by a variety of ingestants and systemic conditions which have in common either a direct $\omega$ action on the surface epithelial cells or an indirect action on the mucous membrane through a reduction in blood 0 flow and inhibition of the normal cellular turnover. ${ }^{1}$ We report a case of gastritis induced by self ingestion of chlorhexidine gluconate $4 \%$ (Hydrex). To our knowledge, this has not previously been reported.

A 72 year old man was admitted to St Charles' Hospital 
for investigation of palpitations and postural hypotension. He also suffered from Parkinson's disease but was well controlled on treatment.

The positive findings on examination included the classical features of Parkinson's disease and a postural drop in blood pressure. The latter quickly resolved on withdrawal of his diuretic therapy. Two days after admission he started vomiting, initially four times on one day and then intermittently over the following 10 days. This usually occurred in the mornings. He was not receiving any new medication.

Fibreoptic gastroscopy showed multiple erosions in the lower part of his stomach and first part of the duodenum. His oesophagus appeared normal. Histology confirmed an active atrophic gastritis with many helicobacter-like organisms present. A full blood count, biochemical screen and liver function tests were all normal. It transpired that every morning he washed himself on the ward with Hydrex. However, he also used this preparation as a mouth wash and then swallowed it. He said that he felt nauseated each time he did this.

He was advised to discontinue this practice and was given ranitidine. He made a full recovery with no further vomiting and repeat endoscopy after 6 weeks revealed resolution of his mucosal erosions with some mild residual antral gastritis.

Chlorhexidine is a bisbiguanide disinfectant which is effective against a wide range of vegetative Gram-positive and Gram-negative bacteria. It is most active at a neutral or slightly acidic pH. Hydrex is a $4 \%$ solution of chlorhexidine gluconate in detergent that is used in pre-operative skin preparation and hand washing. ${ }^{2}$ Skin sensitivity, transient taste disturbance and oral desquamation have occasionally been reported with a variety of preparations of chlorhexidine.

There has been one report of an acute hepatitis and liver necrosis following ingestion of chlorhexidine gluconate in a suicide attempt. ${ }^{3}$ Because it is irritant, it is recommended that it should not be used on the brain, meninges, eyes, middle ear or other sensitive tissues.

There have been no previous reports of chlorhexidineinduced gastritis. This is presumably because the taste and consistency of concentrated preparations of chlorhexidine render them sufficiently unpalatable to discourage ingestion in the majority of cases. We have informed the manufacturers of Hydrex of our unusual observation in the hope that a recurrence of this problem can be avoided by providing a more visible written warning on the container.

\section{S. Roche \\ R. Chinn \\ S. Webb \\ Department of Medicine for the Elderly, St Charles' Hospital, Exmoor Street, London W10, UK.}

\section{References}

1. Shearman, D.J.C. \& Finlayson, N.D. (ed.). Gastritis. In: Diseases of the Gastrointestinal Tract and Liver. Churchill Livingstone, Edinburgh, 1982, pp. 220-225.

2. Reynolds, J.E. (ed.). Martindale. The Extra Pharmacopoeia, 29th Ed. The Pharmaceutical Press, London, 1989, pp. 955957.
3. Massano, G., Ciocatto, E., Rosabianco, C., Vercelli, D., Actis, G.C. \& Verme, G. Striking aminotransferase rise after chlorhexidine self poisoning. Lancet 1982, i: 289.

\section{The cardiovascular effects of beta adrenergic agonist drugs administered by nebulization}

Sir,

I read with interest the report of Flatt et al. ${ }^{1}$ reporting the cardiovascular effects of inhaled bronchodilators. They clearly demonstrate the greater cardiovascular effects of fenoterol $5 \mathrm{mg}$ compared with equal doses of salbutamol and terbutaline. They conclude that this is due to 'greater $\beta 1$ adrenoreceptor stimulation following fenoterol'. However, I think a more satisfactory explanation of their findings is that fenoterol has a greater systemic bioavailability than salbutamol or terbutaline (i.e. more fenoterol is absorbed).

The authors have assumed that the tachycardia and positive inotropic effects of the drugs are primarly due to cardiac $\beta 1$ adrenoreceptor stimulation. In studies of human subjects and tissues, the chronotropic and inotropic effects of salbutamol have been shown to be purely due to cardiac $\beta 2$ adrenoreceptor stimulation. ${ }^{2,3}$ Consequently, all the cardiovascular effects in the study of Flatt et al. could be attributable to cardiac $\beta 2$ adrenoreceptor stimulation and the greater responses seen after fenoterol could be due to greater absorption. Conclusive evidence of greater absorption of fenoterol comes from a previous study published by this group of workers, ${ }^{4}$ where fenoterol by metered dose inhaler produced more hypokalaemia than equal doses of salbutamol. Hypokalaemia induced by $\boldsymbol{\beta}$-agonists is purely due to $\beta 2$ adrenoreceptor stimulation. ${ }^{\mathrm{s}}$

The references cited by Flatt $e$ t al. to show $\beta 1$-mediated effects of fenoterol also assume that chronotropic and inotropic effects are not mediated by $\beta 2$ adrenoreceptors whereas evidence exists to disprove that assumption in the species studied, i.e. in $\operatorname{man}^{2,3}$ and guinea pigs. ${ }^{6}$

Although the findings of differential cardiovascular effects of equal doses of these commonly used bronchodilators are important $I$ think it should be recognized that probably all the cardiovascular effects can be attributed to $\boldsymbol{\beta} 2$ adrenoreceptor stimulation. Consequently in order to avert these important side effects of bronchodilator treatment it would not be necessary to develop an agent with 'greater $\beta 2$ selectivity' but to develop a $\beta 2$ agonist which is less well absorbed.

\section{J.A. Hall \\ Papworth Hospital, Papworth Everard, Cambridge CB3 $8 R E, U K$.}

\section{References}

1. Flatt, A., Crane, J., Purdie, G., Kwong, T., Beasley, R. \& Burgess, C. The cardiovascular effects of beta adrenergic agonist drugs administered by nebulisation. Postgrad Med J 1990, 66: $98-101$.

2. Hall, J.A., Petch, M.C. \& Brown, M.J. Intracoronary injections of salbutamol demonstrate the presence of functional $\beta 2$ adrenoreceptors in the human heart. Circ Res 1989, 65: 546-553. 